\title{
Interdialytic 24-Hours Ambulatory Blood Pressure versus Dialysis Unit Blood Pressure for the Diagnosis of Electrocardiographic-Left Ventricular Hypertrophy in Chronic Hemodialysis Black Patients
}

\author{
Cédric Kabemba Ilunga1, François Bompeka Beka Lepira ${ }^{1 *}$, Jean Robert Rissassi Makulo1, \\ Yves Lubenga ${ }^{2}$, Trésor Mvunzi ${ }^{2}$, Noel Utshudi ${ }^{2}$, Aliocha Nkodila ${ }^{3}$, Vieux Momeme Mokoli ${ }^{1}$, \\ Ernest Kiswaya Sumaili ${ }^{1}$, Nazaire Mangani Nseka ${ }^{1}$, Eleuthère Vita Kintoki ${ }^{2}$
}

\author{
${ }^{1}$ Division of Nephrology, Department of Internal Medicine, University of Kinshasa Hospital, Kinshasa, the Democratic Republic \\ of the Congo \\ ${ }^{2}$ Division of Cardiology, Department of Internal Medicine, University of Kinshasa Hospital, Kinshasa, the Democratic Republic of \\ the Congo \\ ${ }^{3}$ Centre medical cite des Aveugles, Kinshasa, Congo \\ Email: ${ }^{\star}$ lepslepira@yahoo.fr, ${ }^{\star}$ fr.lepira@gmail.com
}

How to cite this paper: Ilunga, C.K., Lepira, F.B.B., Makulo, J.R.R., Lubenga, Y., Mvunzi, T., Utshudi, N., Nkodila, A., Mokoli, V.M., Sumaili, E.K., Nseka, N.M. and Kintoki, E.V. (2019) Interdialytic 24-Hours Ambulatory Blood Pressure versus Dialysis Unit Blood Pressure for the Diagnosis of Electrocardiographic-Left Ventricular Hypertrophy in Chronic Hemodialysis Black Patients. World Journal of Cardiovascular Diseases, 9, 846-856. https://doi.org/10.4236/wjcd.2019.911075

Received: September 19, 2019

Accepted: November 24, 2019

Published: November 27, 2019

Copyright $\odot 2019$ by author(s) and Scientific Research Publishing Inc. This work is licensed under the Creative Commons Attribution International License (CC BY 4.0).

http://creativecommons.org/licenses/by/4.0/

\section{Abstract}

Background and Aim: In hemodialysis patients, 24-hours interdialytic ABPM better detects TOD than dialysis unit blood pressure. Therefore, the present study was aimed to assess the diagnostic performance of 24-hours ABPM vs. dialysis unit BPs for the diagnosis of ECG-LVH in steady state chronic hemodialysis black patients. Methods: From March 31 to September 30, 2018, interdialytic ABPM was performed after a mid-week hemodialysis session for 24 hours using a Spacelab 90207 ABPM monitor in the non-access arm in 45 stable chronic hemodialysis black patients (age $\geq 20$ years, hemodialysis for at least 3 months and informed consent) attending 3 hemodialysis centers in Kinshasa. Ambulatory BP was recorded every 20 minutes during the day (6 AM to $10 \mathrm{PM}$ ) and every 30 minutes during the night (10 PM to 6 AM). ECG-LVH was defined using Cornell product criteria. ROC curve method was used to assess the performance of dialysis unit BPs vs. interdialytic 24-hours ABPM in diagnosing ECG-LVH. P $<0.05$ defined the level of statistical significance. Results: Whatever the method of BP measurement, all the SBP values were related to ECG-LVH with similar AUC and overlapping 95\% $\mathrm{CI}$; however, they were not significantly different from each other. 24-hours interdialytic ambulatory SBP (AUC 0.748; 95\% CI 0.58 - 0.94) had the highest 
area under the curve. Conclusion: The present study showed that although all the two BP measurement methods equally detected ECG-LVH, 24-hours $\mathrm{ABPM}$ tended to have the highest diagnostic performance.

\section{Keywords}

24-Hours ABPM, Dialysis Unit BPs, ECG-LVH, Diagnostic Performance, Chronic Hemodialysis, Black Africans

\section{Introduction}

Hypertension, a common clinical finding among patients with chronic kidney disease (CKD), often remains poorly controlled in maintenance hemodialysis (MHD) and is associated with an increased risk for cardiovascular (CV) events [1]. Therefore, accurate and reliable measurement of blood pressure (BP) in this specific group of patients is of diagnostic, prognostic and therapeutic importance [1]. In this regard, the relationship between BP and CV outcomes in MHD has been reported to depend on the setting and the technique of BP measurement [1]. As the variability of BP within the patient at least as great as variability seen between patients [2], BP obtained routinely using oscillometric method in the dialysis unit without attention to details that differ strikingly from BP obtained using standardized methods [1]. However, BP obtained in the dialysis unit (peridialytic BP), even if obtained by standardized methods, agrees poorly with interdialytic ambulatory BP (ABP) [3] [4] [5], and cannot be used to accurately predict the presence or absence of left ventricular hypertrophy (LVH) [6]. In contrast, $\mathrm{BP}$ obtained outside the dialysis unit, whether obtained by $\mathrm{ABP}$ measurement (ABPM) or home $\mathrm{BP}$, is more useful and accurate to diagnose LVH [6]. It appears thus rationale for each setting to evaluate the relationship between inside and outside BP measurement and intermediate end-point such as LVH as well final end-points such as mortality in MHD patients. Unfortunately, most studies on this topic have been conducted out-of sub-Saharan Africa highlighting the need for data from this region of the world where the access to renal replacement therapy has substantially improved. In the Democratic Republic of the Congo (DRC), a recent study has reported an inverse relationship between peridialytic BP and mortality in MHD patients with stroke [7]. However, the predictive value of peri-dialytic BP compared to 24-hour ABPM for the diagnosis of LVH in steady state MHD patients has not yet been evaluated. Therefore, the aim of the present study was to assess the predictive value of peridialytic BPs and 24-hour ABPM for the diagnosis of eletrocardiographic (ECG)-LVH in steady state MHD patients in Kinshasa, the DRC.

\section{Patients and Methods}

\section{- Participants}

From March 31 to September 30, 2018, patients 20 years or older who had 
been on maintenance hemodialysis (MHD) for more than 3 months and were dialyzed 2 or 3 times a week in three dialysis units in Kinshasa, DR Congo (University of Kinshasa Hospital, Ngaliema Medical Center and Medical Center of Kinshasa) were consecutively enrolled in a cross-sectional on the predictive value of dialysis unit BPs (predialysis, intradialysis and postdialysis BPs) and interdialytic 24-hours ambulatory BP monitoring (ABPM) for the diagnosis of electrocardiographic-left ventricular hypertrophy (ECG-LVH). Inclusion criteria were age $\geq 20$ years, regular hemodialysis for at least 3 months and informed consent. Patients with chronic atrial fibrillation (AF) or body mass index (BMI) $\geq 40 \mathrm{Kg} / \mathrm{m}^{2}$ were excluded. Patient's medical records were used to collect data on past medical history, sociodemographic (age, gender, profession, marital status, financial support), dialysis (type of vascular access, type of dialysis, current month's mean interdialytic weight gain and KT/V, number of sessions per week, dialysate sodium profile) and biological parameters (last month's blood urea nitrogen, serum creatinine, serum albumin, total and ionized calcium, phosphorous) as well as current treatment. The study was approved by the Ethical Committee of Kinshasa School of Public Health and all patients gave written informed consent.

\section{- Measurements}

Peridialysis (pre- and postdialysis BPs) BP measurement was obtained by trained dialysis unit staff using a validated automated sphygmomanometer OMRON MIT5 Connect with patients at a sitting position, within 30 minutes prior to and following the dialysis session on the non-fistula arm or the non-dominant arm for patients using catheters. Three readings 1 to 3 minutes apart were recorded after the patient had been resting in a quiet room for at least 5 minutes; the average of the two last readings was used as the standard BP value for the present study. Intradialysis BP recordings were obtained by the dialysis unit staff using the sphygmomanometer equipped with hemodialysis machines without a specified technique. All BP recordings were averaged over two weeks surrounding the ambulatory BP measurement. Thus, depending on the number of session a weak ( 2 or 3 sessions a week), each patient had 4 or 6 predialysis, postdialysis $\mathrm{BP}$ recordings, respectively, to provide routine dialysis unit.

Interdialysis 24-hours ambulatory BP measurement (ABPM) was performed after a mid-week hemodialysis session for 24 hours using a GIMA ABPM PULSE RATE monitor (Gima Spa, Milano, Italia) in the non-access arm. Ambulatory $\mathrm{BP}$ was recorded every 20 minutes during the day (6 AM to $10 \mathrm{PM}$ ) and every 30 minutes during the night (10 PM to $6 \mathrm{AM}$ ). Patients were instructed to keep their arm immobile during measurement and follow their daily activity. Awake and sleep readings were calculated for each patient by self-reported sleep and wake times by means of a diary. Patients with $<70 \%$ recording were excluded for the analysis.

\section{- Outcome}

Resting ECG records lasting less than 3 months were retrieved from patients' medical files whereas it was performed in patients with ECG records lasting 
more than 3 months. Left ventricular mass (LVM) was estimated using Cornell product index; ECG-LVH was defined LMV > $2.440 \mathrm{~mm} . \mathrm{sec}$ [8].

\section{- Statistical analyses}

Baseline characteristics were summarized as mean (standard deviation) or median (interquartile range) for continuous variables and as absolute (n) and relative (in \%) frequencies for categorical variables. The comparison of means of dialysis unit $\mathrm{BP}$ and 24-hours $\mathrm{ABP}$ was performed using one way analysis of variance (ANOVA). Receiver operating characteristic (ROC) analysis was conducted for evaluating the predictive performance (Area Under the Curve, AUC) of 24-hours ABP vs dialysis unit BP and for the diagnosis of ECG-LVH. P value $<0.05$ defined the level of statistical significance.

\section{Results}

\section{- General and hemodialysis characteristics of the study population}

Of the 62 patients eligible for the study, 17 of them were excluded [non-consent: 2, dialysis arrest: 1 , hemodynamic instability: 5 , death during the study: 4 , travel abroad: 3 and non-valid ABP recordings (arrhythmia: 1, intolerance: 1)]. Finally, 45 patients (31 men and 14 women) constituted the sample population of the present study (Table 1), their mean age and body mass index (BMI) was $59.1 \pm$ 12.3 years and $24.4 \pm 3.9 \mathrm{Kg} / \mathrm{m}^{2}$, respectively. Hypertension and diabetes as the initial underlying kidney disease were reported by 25 (55.6\%) and 13 (28.9) patients, respectively. Hypertension with a mean duration of $12.1 \pm 3.1$ years was present in 44 (97.8\%) patients. Calcium channel blockers (CCB) and diuretics were the antihypertensive drug classes reported by 39 (88.6\%) and 29 (65.9\%) patients, respectively. ECG-LVH using Cornell product index was present in 11 (25\%) patients.

Hemodialysis parameters of the study population are depicted in Table 1 . The mean HD vintage was $34.3 \pm 10.8$ months with patients having twice and thrice a week dialysis session, respectively. The majority of patients $(n=28)$ had a catheter as vascular access and were receiving either $\operatorname{HDF}(n=22)$ or the combination of $\mathrm{HDF}$ and $\mathrm{HD}(\mathrm{n}=17)$. Average levels of KT/V, IDWG and RD were $1.2 \pm 0.2$, $1.9 \pm 0.4 \mathrm{Kg}$ and $338.8 \pm 36.6 \mathrm{~mL} /$ day, respectively. Nearly all patients were receiving EPO $(n=100)$ and IV iron $(n=41)$. The majority of patients $(n=35)$ had a secured financial support. Average levels of $\mathrm{Hb}, \mathrm{BUN}$, creatinine, and albumin were $11.1 \pm 1.9 \mathrm{~g} / \mathrm{dL}, 111.4 \pm 46.5 \mathrm{mg} / \mathrm{dL}$ and $9.2 \pm 3.6 \mathrm{mg} / \mathrm{dL}$, respectively (Table 2).

\section{- Dialysis unit and interdialytic 24-h ambulatory BP measurements}

Table 3 summarizes average dialysis unit and 24-h ABP levels of the study population. Interdialytic 24-h ABP components tended to be in average lower than that of dialysis unit BPs; however, the difference was statistically significant $(\mathrm{p}=0.014)$ only for SBP.

\section{- Dialysis unit BPs vs. 24-h ABPM for the diagnosis of ECG-LVH}

Figure 1 compares ROC curves of dialysis unit SBPs vs 24-h interdialytic 
ambulatory SBP for the diagnosis of ECG-LVH. Whatever the method of BP measurement, all the SBP values were related to ECG-LVH with overlapping 95\% CI; however, they were not significantly different from each other. Twenty four-hours interdialytic ambulatory SBP (AUC 0.748; 95\% CI 0.58 - 0.94) had

Table 1. Demographic, clinical and biological characteristics of the study population.

\begin{tabular}{|c|c|}
\hline Variables & All $(n=45)$ \\
\hline Age, years & $59.1 \pm 12.3$ \\
\hline \multicolumn{2}{|l|}{ Gender, n (\%) } \\
\hline M & $31(68.8)$ \\
\hline $\mathrm{F}$ & $14(31.2)$ \\
\hline \multicolumn{2}{|c|}{ Initial Kidney disease, n (\%) } \\
\hline Hypertension & $25(55.6)$ \\
\hline Diabetes & $13(28.9)$ \\
\hline CGN & $8(17.8)$ \\
\hline Others & $4(8.9)$ \\
\hline BMI, $\mathrm{Kg} / \mathrm{m}^{2}$ & $24.4 \pm 3.9$ \\
\hline Hypertension, $\mathrm{n}(\%)$ & $44(97.8)$ \\
\hline DHT, years & $12.1 \pm 3.1$ \\
\hline \multicolumn{2}{|c|}{ Antihypertensive drugs, n (\%) } \\
\hline $\mathrm{CCB}$ & $39(86.7)$ \\
\hline Diuretic & $29(64.4)$ \\
\hline ACEIs & $14(31.8)$ \\
\hline Betablockers & $12(26.6)$ \\
\hline ARBs & $9(20.0)$ \\
\hline Others & $9(20.0)$ \\
\hline ECG-LVH, n (\%) & $11(24.4)$ \\
\hline Hemoglobin, g/dL & $11.1 \pm 1.9$ \\
\hline Hematocrit, \% & $33.2 \pm 5.9$ \\
\hline BUN, mg/dL & $111.4 \pm 46.5$ \\
\hline Creatinine, $\mathrm{mg} / \mathrm{dL}$ & $9.2 \pm 3.6$ \\
\hline Albumin, g/dL & $36.8 \pm 13.9$ \\
\hline Sodium, mmol/L & $128.9 \pm 29.6$ \\
\hline Potassium, $\mathrm{mmol} / \mathrm{L}$ & $5.4 \pm 1.6$ \\
\hline Calcium, mmol/L & $2.13 \pm 0.31$ \\
\hline
\end{tabular}

Data are expressed as mean \pm standard deviation, absolute (n) and relative (in percent) frequencies. Abbreviations: M, male F, female CGN, chronic glomerulonephritis BMI, body mass index DHT, duration of hypertension CCB, calcium channel blocker ACEIs, angiotensin converting enzyme inhibitors $\mathrm{ARBs}$, angiotensin type 1 receptor blockers ECG-LVH, electrocardiographic-left ventricular hypertrophy BUN, blood urea nitrogen. 
Table 2. Hemodialysis parameters of the study population.

\begin{tabular}{|c|c|}
\hline Variables & All $(n=45)$ \\
\hline \multicolumn{2}{|l|}{ Type, n (\%) } \\
\hline HD & $6(13.3)$ \\
\hline $\mathrm{HDF}$ & $22(48.9)$ \\
\hline $\mathrm{HD} / \mathrm{HDF}$ & $17(37.8)$ \\
\hline Dialysis duration, mo & $34.3 \pm 10.8$ \\
\hline \multicolumn{2}{|l|}{ Vascular access, n (\%) } \\
\hline Catheter & $28(62.2)$ \\
\hline $\mathrm{AVF}$ & $17(37.8)$ \\
\hline $\mathrm{KT} / \mathrm{V}$ & $1.2 \pm 0.2$ \\
\hline IDWG, Kg & $1.9 \pm 0.4$ \\
\hline $\mathrm{RD}, \mathrm{mL} /$ day & $338.8 \pm 36.6$ \\
\hline$>500$ & $6(20.7)$ \\
\hline $200-400$ & $12(41.4)$ \\
\hline$<200$ & $11(37.9)$ \\
\hline EPO, n (\%) & $45(100)$ \\
\hline EPO dosing, IU/Kg/week & $11,187.8 \pm 2450.5$ \\
\hline IV iron therapy, n (\%) & $41(91.1)$ \\
\hline IV Iron dosing, mg/week & $130.6 \pm 46.7$ \\
\hline \multicolumn{2}{|l|}{ Financial support, n (\%) } \\
\hline Patient/family & $8(17.8)$ \\
\hline Private and public entreprises & $35(77.8)$ \\
\hline Government & $2(4.4)$ \\
\hline
\end{tabular}

Data are expressed as mean \pm standard deviation, absolute (n) and relative (in percent) frequencies. Abbreviations: HD, hemodialysis HDF, hemodiafiltration AVF, arteriovenous fistulae KT/V, dialysis efficacy EPO, erythropoietin IU, international unit IV intravenous.

Table 3. Average levels of dialysis unit blood pressure and interdialytic 24-hours ambulatory blood pressure of the study population $(n=45)$.

\begin{tabular}{cccccc}
\hline Variable & PreHD & PostHD & IntraHD & 24 h-ABPM & p \\
\hline SBP, mmHg & $152.3 \pm 19.4$ & $149.2 \pm 18.6$ & $153.4 \pm 22.0$ & $141.1 \pm 17.8$ & 0.014 \\
DBP, mmHg & $88.5 \pm 13.6$ & $86.5 \pm 10.9$ & $95.1 \pm 10.4$ & $82.1 \pm 12.5$ & 0.705 \\
PP, mmHg & $63.8 \pm 11.9$ & $62.7 \pm 14.5$ & $58.3 \pm 11.0$ & $59.0 \pm 11.8$ & 0.957 \\
MAP, mmHg & $109.8 \pm 14.7$ & $107.4 \pm 12.1$ & $114.5 \pm 16.0$ & $101.8 \pm 13.4$ & 0.405 \\
\hline
\end{tabular}

Data are expressed as mean \pm standard deviation Abbreviations: PreHD, prehemodialysis PostHD, Posthemodialysis IntrHD, intrahemodialysis SBP, systolic blood pressure DBP, diastolic blood pressure PP, pulse pressure MAP, mean arterial blood pressure. 


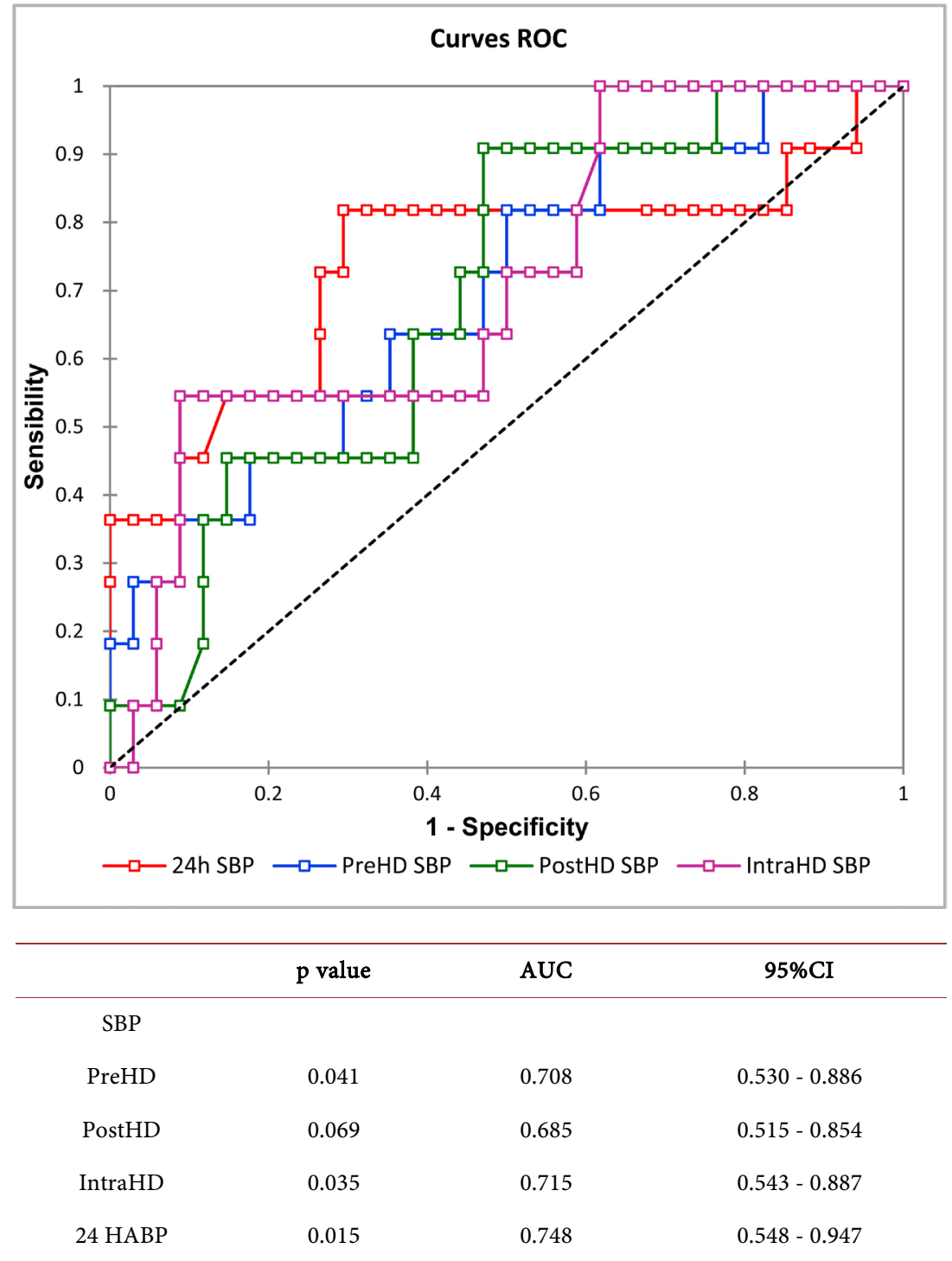

Figure 1. Receiver operated characteristic (ROC) curves and area under the curve (AUC) of interdialytic 24-hour ambulatory systolic BP vs dialysis unit systolic blood pressure (BP) vs for the diagnosis of ECG-LVH. (Abbreviations: PreHD, pre-hemodialysis, post-HD, post-hemodialysis, intra-HD, intra-hemodialysis, 24 HABPM, 24-hours ambulatory blood pressure).

the highest area under the curve followed by IntraHD (AUC 0.715; 95\% CI 0.58 0.94), PreHD (AUC 0.708; 95\% CI 0.53 - 0.88) and PostHD (AUC 0.685; 95\% CI 0.51 - 0.85) SBPs, respectively.

\section{Discussion}

The main findings of the present study are as follows. First, average levels of 24-h interdialytic ambulatory BP levels were lower compared with those obtained with dialysis unit measurements. Second, whatever the BP measurement method, all SBP predicted equally ECG-LVH without significant difference from each other; 24-h interdialytic ambulatory SBP had the highest area under the 
curve followed by intradialytic SBP.

24-h interdialytic ambulatory BP levels were in average lower than that obtained by dialysis unit BP measurements. This finding is consistent with that of Argawal et al. [6] who reported in a meta-analysis that pre- and postdialysis blood pressures are imprecise estimates of interdialytic blood pressure. They found that predialysis BP overestimated ambulatory BP whereas postdialysis BP underestimated it. They linked the higher predialysis measurements to increased intravascular volume, withholding antihypertensive medications just before treatment, white coat effect, and lack of standardized measurements.

Whatever the BP measurement method used, all SBP equally predicted ECG-LVH with 24-h interdialytic ambulatory SBP having the highest AUC. Consistent with our finding, Agarwal et al. [9] already reported that using all BP values pre- and postdialysis measured a mid-week dialysis may serve as a more useful tool to estimate interdialytic ambulatory BP. Although all BP measurement methods have already been reported to predict hypertension and related target organ damage (TOD) such as LVH in chronic hemodialysis patients, their sensitivity and specificity condition their diagnostic performance [9]. In this regard, ABPM is considered as the gold standard for the diagnosis and management of hypertension in chronic hemodialysis patients [9] [10]. The superiority of ABPM over dialysis unit BP measurement is thought to rely upon the provision of a greater number of measurements and a more accurate reflection of the patient's BP burden overtime [9]. In addition, whereas dialysis unit BP is influenced by the white-coat effect, the latter is eliminated with the use of ABPM [11]. Agarwal et al. [6] found that dialysis unit BP measurements were weak correlates of LVH and, whatever the BP measurement technique used, DBP was not associated with LVH. Of note, SBP outside the dialysis unit was a stronger correlate of echocardiographic [6] [12] as well as ECG-LVH [13] compared with dialysis unit BP. In an attempt to improve the diagnostic performance of dialysis unit BP measurements, Rahman et al. [13] found that standardizing the technique of blood pressure measurement was associated with statistically lower preand postdialysis BPs. The authors stress out that the magnitude of the measurement errors should attenuate the difference between peridialysis BP and ambulatory BP. However, this improvement remains still a matter of controversy and debate since it was not found in some studies, like ours, that used standardized BP [6] [14].

Intradialytic BP had the second highest area under the curve for diagnosing ECG-LVH in the present study. Another way to improve diagnostic accuracy of peridialysis $\mathrm{BP}$ measurements is to consider intradialytic $\mathrm{BP}$ recordings obtained via an automatic cuff attached to the HD machine [9] [15] [16]. Indeed, the median intradialytic BP has been suggested to serve as useful tool to assess hypertension and related TOD and may therefore be used a bedside tool to predict interdialytic ambulatory BP. A mid-week intradialytic BP $\geq 140 / 90 \mathrm{mmHg}$ has sensitivity and specificity that exceeds pre- or postdialysis BP measurements. 
However, this method is of last resort because better methods are available to evaluate hypertension and related TOD in hemodialysis patients. In addition, when diagnosing hypertension using $\mathrm{ABPM}$ as a gold standard, average intradialytic BP considered together with peridialytic BP has greater diagnostic value compared to peridialytic BP recordings alone [11]. It is possible that intradialytic $\mathrm{BP}$ correlates better with ambulatory BP over peridialytic BP because the latter, similar to interdialytic BP, samples the patient over a range of extracellular fluid volume and uremic states during the condensed time span of the HD session [11].

The interpretation of the results of the present study should take into account some limitations. First, the cross-sectional nature of the study precludes the establishment of any temporal relationship between the variables of interest. Second, the relatively small study sample size did not allow much power to statistical tests to detect potential association between the variables of interest. Third, single peridialytic BP and 24-h ambulatory BP measurements could have led to under- or overestimation of average BP levels. Fourth, electrocardiogram a less sensitive method was used to assess left ventricular mass and left ventricular hypertrophy.

\section{Conclusion}

Although all BP measurement methods equally diagnosed ECG-LVH among the present case series, 24-h interdialytic ambulatory BP measurement had the highest diagnostic performance compared to dialysis unit BP measurements.

\section{Acknowledgements}

The authors gratefully thank the medical staff of all the participating ICUs (Professor Dr. Jean Robert Rissassi Makulo, Head of University of Kinshasa Hospital; Prof Dr. Eleuthère Vita Kintoki, Head of the Division of Cardiology/University of Kinshasa Hospital; Dr. Nyakabasa, Head of Ngaliema Medical Center; Dr. Rodolph, Head of Kinshasa Medical Center, Dr. Justine Busangu Bukabau, Head Medical Staff of Kinshasa Medical Center) for their outstanding help during the conduct of the present study. The authors would like also to express their deepest gratitude to all the participants who facilitate by their informed consent the implementation of the present study.

\section{Authors Contribution}

CKI collected data, participated in data analysis and reviewed the manuscript.

FBBL conceived the study, participated in data analysis and drafted the manuscript.

YL participated in data collection and reviewed the manuscript.

TM participated in data collection and reviewed the manuscript.

NU participated in data collection and reviewed the manuscript

AN conducted statistical analysis of data and reviewed the manuscript. 
VMM reviewed the manuscript.

EKS participated in statistical analysis of data and reviewed the manuscript.

NMN reviewed the manuscript.

EVK reviewed the manuscript.

\section{Conflicts of Interest}

The authors declare no conflicts of interest regarding the publication of this paper.

\section{References}

[1] Agarwal, R. (2010) Blood Pressure and Mortality among Hemodialysis Patients. Hypertension, 55, 762-768. https://doi.org/10.1161/HYPERTENSIONAHA.109.144899

[2] Rohrscheib, M.R., Myers, O.B., Servilla, K.S., et al. (2008) Age-Related Blood Pressure Patterns and Blood Pressure Variability among Hemodialysis Patients. Clinical Journal of the American Society of Nephrology, 3, 1407-1414. https://doi.org/10.2215/CJN.00110108

[3] Agarwal, R. and Lewis, R.R. (2001) Prediction of Hypertension in Chronic Hemodialysis Patients. Kidney International, 60, 1982-1989.

https://doi.org/10.1046/j.1523-1755.2001.00997.x

[4] Agarwal, R., Andersen, M.J., Bishu, K. and Saha, C. (2006) Home Blood Pressure Monitoring Improves the Diagnosis of Hypertension in Hemodialysis Patients. Kidney International, 69, 900-906. https://doi.org/10.1038/sj.ki.5000145

[5] Agarwal, R., Peixoto, A.J., Santos, S.F. and Zoccali, C. (2006) Pre and Post Dialysis Blood Pressures Are Imprecise Estimates of Interdialytic Ambulatory Blood Pressure. Clinical Journal of the American Society of Nephrology, 1, 389-398. https://doi.org/10.2215/CJN.01891105

[6] Agarwal, R., Brim, N.J., Mahenthiran, J., Andersen, M.J. and Saha, C. (2006) Out-of-Hemodialysis-Unit Blood Pressure Is a Superior Determinant of Left Ventricular Hypertrophy. Hypertension, 47, 62-68.

https://doi.org/10.1161/01.HYP.0000196279.29758.f4

[7] Nlandu, Y., Lepira, F., Makulo, J.R., et al. (2017) Reverse Epidemiology of Elevated Blood Pressure among Chronic Hemodialysis Black Patients with Stroke: A Historical Cohort Study. BMC Nephrology, 18, 277. https://doi.org/10.1186/s12882-017-0697-0

[8] Casale, P.N., Devereux, R.B., Kligfield, P., et al. (1985) Electrocardiographic Detection of Left Ventricular Hypertrophy Development and Prospective Validation of Improved Criteria. Journal of the American College of Cardiology, 6, 572-580.

[9] Agarwal, R., Flynn, J., Pogue, V., et al. (2014) Assessment and Management of Hypertension in Patients on Dialysis. Journal of the American Society of Nephrology, 25, 1630-1646. https://doi.org/10.1681/ASN.2013060601

[10] Agarwal, R. (2010) Managing Hypertension Using Home Blood Pressure Monitoring among Hemodialysis Patients. A Call for Action. Nephrology Dialysis Transplantation, 25, 1766-1711. https://doi.org/10.1093/ndt/gfq171

[11] Georgianos, P.I. and Agarwal, R. (2017) Blood Pressure in Hemodialysis: Targets? Current Opinion in Nephrology and Hypertension, 26, 523-529.

https://doi.org/10.1097/MNH.0000000000000359 
[12] Nassiri, A.A., Lotfollahi, L., Behzadonia, N., et al. (2018) Blood Pressure Measurement and Left Ventricular Mass Index in Hemodialysis. Comparison of Several Methods. Iranian Journal of Kidney Diseases, 12, 185-189.

[13] Rahman, M., Griffin, V., Kumar, A., et al. (2002) A Comparison of Standardized versus "Usual" Blood Pressure Measurements in Hemodialysis Patients. American Journal of Kidney Diseases, 39, 1226-1230. https://doi.org/10.1053/ajkd.2002.33395

[14] Khangura, J., Culleton, B.F., Manns, B.J., et al. (2010) Association between Routine and Standardized Blood Pressure Measurements and Left Ventricular Hypertrophy among Patients on Hemodialysis. BMC Nephrology, 11, 13.

https://doi.org/10.1186/1471-2369-11-13

http://www.biomedcentral.com/1471-2369/11/13

[15] Yoon, I.C., Choi, H.M. and Oh, D.J. (2017) Which Dialysis Unit Blood Pressure Is the Most Accurate for Predicting Home Blood Pressure in Patients Undergoing Hemodialysis? The Korean Journal of Internal Medicine, 32, 117-124. https://doi.org/10.3904/kjim.2015.278

[16] Van Buren, P.N., Toto, R. and Inrig, J.K. (2011) Intradialytic Hypertension and the Association with Interdialytic Ambulatory Blood Pressure. Clinical Journal of the American Society of Nephrology, 6, 1684-1691.

https://doi.org/10.2215/CJN.11041210 